\title{
Co-expression of neuronal intermediate filaments, peripherin and $\alpha$-internexin in human well-differentiated endocrine neoplasms (carcinoid tumors) of the appendix
}

\author{
MITSUAKI ISHIDA ${ }^{1}$, RYOJI KUSHIMA ${ }^{2}$, MARIE BREVET $^{3,4}$, DENIS CHATELAIN $^{4}$ and HIDETOSHI OKABE ${ }^{1,2}$ \\ ${ }^{1}$ Department of Clinical Laboratory Medicine, ${ }^{2}$ Division of Diagnostic Pathology, and ${ }^{3}$ Department of Anatomy, \\ Shiga University of Medical Science, Shiga, Japan; ${ }^{4}$ Department of Pathology, \\ Medical University and Hospital, Amiens, France
}

Received November 9, 2007; Accepted January 12, 2008

\begin{abstract}
The rectum and appendix are the two major sites of well-differentiated endocrine neoplasms (carcinoid tumors) in the lower gastrointestinal tract. Previously, we reported the consistent expression of peripherin in rectal well-differentiated endocrine neoplasms without metastases. However, its expression has not as yet been examined in appendiceal welldifferentiated endocrine neoplasms. The aim of our present study was to clarify whether peripherin, a type III neuronal intermediate filament, and $\alpha$-internexin, a type IV neuronal intermediate filament, are expressed in appendiceal welldifferentiated endocrine neoplasms. Other endocrine markers were also examined and compared with the findings from the rectal well-differentiated endocrine neoplasms. The analyses were carried out by immunohistochemical methods using 12 formalin-fixed and paraffin-embedded appendiceal welldifferentiated endocrine neoplasms. In all the neoplasms examined, diffuse immunoreactivity of peripherin was observed. In addition, immunoreactivity of $\alpha$-internexin, which was frequently co-expressed with peripherin, was found in all appendiceal cases. Chromogranin A and neural cell adhesion molecule expression was found in all appendiceal tumors, and serotonin was also frequently expressed (83\%, 10/12 cases). Incidences of the expression of these three markers were much higher in the appendiceal than in the rectal cases. Peripherin expression is a common feature of appendiceal and rectal welldifferentiated endocrine neoplasms, but the manner of neural marker expression is different depending on the site of origin. It is uncertain whether the expression of peripherin and/or
\end{abstract}

Correspondence to: Dr Hidetoshi Okabe, Department of Clinical Laboratory Medicine and Division of Diagnostic Pathology, Shiga University of Medical Science, Tsukinowa-cho, Seta, Otsu, Shiga 520-2192, Japan

E-mail: okabe@belle.shiga-med.ac.jp

Key words: well-differentiated endocrine neoplasm, carcinoid tumor, peripherin, $\alpha$-internexin, appendix $\alpha$-internexin is present in the well-differentiated endocrine neoplasms of other organs; further analysis is required to clarify this issue.

\section{Introduction}

Neurons have several different intermediate filaments, such as neurofilament, peripherin and $\alpha$-internexin (1-4). These intermediate filaments are involved in the development and maintenance of various neurons, and their distribution is not always the same (1-5). They are frequently expressed in the central and peripheral neuroblastomas and medulloblastomas (6-8), but not in the majority of non-neural neoplasms. Peripherin belongs to a group of type III intermediate filaments. It is expressed throughout the lifetime of a subgroup of peripheral neurons, from their early developmental stage $(1,2)$, and is frequently found in cutaneous endocrine carcinomas (Merkel cell carcinomas) (9) in addition to neuroblastomas (6). Recently, we demonstrated the presence of consistent peripherin expression in rectal well-differentiated endocrine neoplasms (carcinoid tumors) without metastases (10). $\alpha$-internexin, a $66-\mathrm{kDa}$ type IV intermediate filament, is quite abundantly expressed in various kinds of central and peripheral neurons from early development $(3,4)$, and is frequently demonstrated in medulloblastomas (7) and neuroblastomas (8). However, there have been no reports on an immunohistochemical analysis of this intermediate filament in gastrointestinal endocrine neoplasms. Here, we report the results of immunohistochemical analyses of peripherin and $\alpha$-internexin expression in appendiceal well-differentiated endocrine neoplasms. Several endocrine markers were also examined for comparison with the findings from the rectal well-differentiated endocrine neoplasms.

\section{Materials and methods}

Tissue specimens of appendiceal well-differentiated endocrine neoplasms. Materials consisted of 12 formalinfixed and paraffin-embedded tissue specimens of consecutive appendiceal well-differentiated endocrine neoplasms (carcinoid tumors) obtained from 11 patients (cases 1-11) at Amiens University Hospital and 1 patient (case 12) at the Shiga 
Table I. Clinicopathological and immunohistochemical features of appendiceal well-differentiated endocrine neoplasms (carcinoid tumors).

\begin{tabular}{|c|c|c|c|c|c|c|c|c|c|}
\hline Case & Age/sex & Size $(\mathrm{mm})$ & Peripherin & Internexin & Serotonin & NCAM & Synaptophysin & Chromogranin A & Ki-67 index \\
\hline 1 & $69 / \mathrm{M}$ & 15 & $3+$ & $3+$ & $3+$ & $3+$ & $3+$ & $3+$ & $<1 \%$ \\
\hline 2 & $14 / \mathrm{M}$ & 4 & $3+$ & $3+$ & $3+$ & $3+$ & $3+$ & $3+$ & $<1 \%$ \\
\hline 3 & $45 / F$ & 20 & $3+$ & $3+$ & $3+$ & $3+$ & $3+$ & $3+$ & $<1 \%$ \\
\hline 4 & $41 / \mathrm{F}$ & 5 & $3+$ & $3+$ & $3+$ & $3+$ & $3+$ & $3+$ & $<1 \%$ \\
\hline 5 & $28 / \mathrm{M}$ & 3 & $3+$ & $2+$ & 0 & $1+$ & $3+$ & $2+$ & $<1 \%$ \\
\hline 6 & $74 / \mathrm{M}$ & 2 & $3+$ & $3+$ & 0 & $2+$ & $3+$ & $3+$ & $<1 \%$ \\
\hline 7 & $44 / F$ & 15 & $3+$ & $2+$ & $3+$ & $3+$ & $3+$ & $3+$ & $<1 \%$ \\
\hline 8 & $32 / \mathrm{F}$ & 13 & $3+$ & $1+$ & $3+$ & $3+$ & $3+$ & $3+$ & $<1 \%$ \\
\hline 9 & $25 / \mathrm{F}$ & 4 & $3+$ & $2+$ & $3+$ & $2+$ & $3+$ & $3+$ & $<1 \%$ \\
\hline 10 & $18 / \mathrm{M}$ & 9 & $3+$ & $3+$ & $3+$ & $3+$ & $3+$ & $3+$ & $<1 \%$ \\
\hline 11 & $44 / \mathrm{M}$ & 9 & $3+$ & $2+$ & $3+$ & $1+$ & $3+$ & $3+$ & $<1 \%$ \\
\hline 12 & $66 / \mathrm{M}$ & 10 & $3+$ & $3+$ & $3+$ & $2+$ & $3+$ & $3+$ & $<1 \%$ \\
\hline
\end{tabular}

0 , no positive cells; $1+$, positive cells $<30 \%$; +2 , positive cells range from 31 to $69 \%$; and +3 , positive cells $>70 \%$.

University of Medical Science Hospital between 2000 and 2006 (Table I). All specimens were operative cases, and the size of each tumor was recorded according to its maximum dimension after formalin fixation. The median age of the patients (7 men and 5 women) was 41.7 years (range 14-74). All specimens were re-evaluated by at least two diagnostic pathologists according to the criteria described in the World Health Organization Classification of Tumors, Pathology and Genetics of Tumors of the Digestive System (11), and Histological Typing of Endocrine Tumors (12).

Elastica van Gieson staining was performed in addition to hematoxylin and eosin staining for the evaluation of angioinvasion.

Immunohistochemistry. Deparaffinized $3-\mu \mathrm{m}$ sections of the specimens were processed for the LSAB system (Dako Japan Co. Ltd., Kyoto, Japan) using the following primary antibodies: peripherin monoclonal mouse antibody (diluted 1:100; PJM50; Novocastra Laboratories Ltd., Newcastle upon Tyne, UK), $\alpha$-internexin monoclonal mouse antibody (diluted 1:50; 2E3; Lab Vision Corporation, CA, USA), serotonin monoclonal mouse antibody (diluted 1:100; 5HT-H209; Dako), Ki-67 monoclonal mouse antibody (diluted 1:50; MM1; Novocastra), NCAM (neural cell adhesion molecule; CD56) monoclonal mouse antibody (diluted 1:100; CD564; Novocastra), synaptophysin monoclonal mouse antibody (diluted 1:200; 27G12; Novocastra) and chromogranin A monoclonal mouse antibody (diluted 1:50; LK2H10; Novocastra). Antigen retrieval by 20 min of incubation in a microwave oven at $500 \mathrm{~W}$ with $0.01 \mathrm{M}$ citrate ( $\mathrm{pH}$ 6.0) was performed before the application of the primary antibodies to peripherin, $\alpha$-internexin, Ki-67, NCAM, synaptophysin and chromogranin A. Pronase $(0.05 \%)$ digestion for $15 \mathrm{~min}$ at $37^{\circ} \mathrm{C}$ was performed for anti-serotonin. The sections were then washed with $0.1 \mathrm{M}$ phosphate-buffered saline ( $\mathrm{pH} 7.4$ ) and bathed in $3 \% \mathrm{H}_{2} \mathrm{O}_{2}$ in methanol for $20 \mathrm{~min}$ at room temperature to quench endogenous peroxidase activity.
Subsequently, primary antibodies were applied to the sections, which were then incubated in a humidified chamber at $4^{\circ} \mathrm{C}$ overnight. After three washing steps in phosphate-buffered saline, sections were incubated with biotinylated secondary antibody for $20 \mathrm{~min}$ at room temperature and then sequentially stained using a $0.1 \%$ peroxidase-conjugated streptavidin solution, according to the manufacturer's instructions. Finally, sections were incubated in 3,3'-diaminobenzidine tetrahydrochloride (DAB; Nichirei) for a few minutes and then counter-stained with hematoxylin. Immunohistochemical staining of peripherin and other markers was carried out in serial sections to evaluate the correlation between marker distributions.

Evaluation of immunoreactivity. Immunohistochemical findings were verified by control studies. Negative controls consisted of slides processed without the addition of primary antibody. The specificity of the immunoreactivity to individual antibodies was evaluated by the inner and outer positive controls. Non-neoplastic appendiceal mucosal endocrine cells within the tumor tissue slides were used as inner controls for the immunoreactivity of serotonin, synaptophysin and chromogranin $\mathrm{A}$, and submucosal ganglia and nerve plexus in the muscularis propria were used as a positive control for peripherin, $\alpha$-internexin and NCAM. Sections of dorsal root ganglia mounted on the slides were used as the external positive control for peripherin, and sections of neurons of the cerebrum were used as the external positive controls for $\alpha$-internexin. Lymph node sections were used as the external positive control for Ki-67.

Ki-67 labeling indices were evaluated by counting the percentage of positive cells in 1,000 tumor cells. The immunoreactivity of other markers was classified according to the following four categories: 0 , no positive cells in the tumor; $1+$, positive cells comprising $<30 \%$ of the tumor; $2+$, positive cells ranging from 31 to $69 \% ; 3+$, positive cells comprising $>70 \%$. 


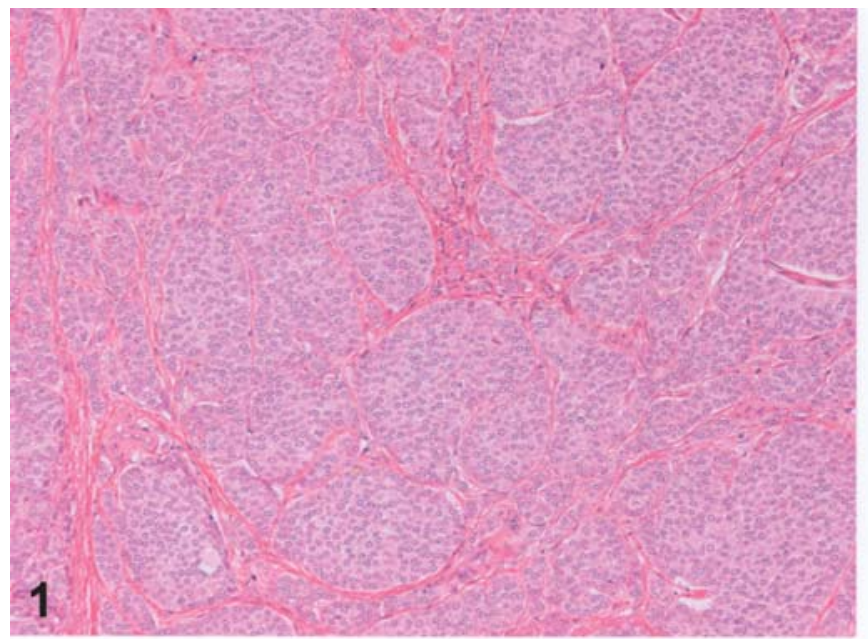

Figure 1. Typical well-differentiated endocrine neoplasm (carcinoid tumor) of the appendix with insular growth of uniform neuroendocrine cells (case 4). $\mathrm{H} \& \mathrm{E}$ (original magnification, $\mathrm{x} 100$ ).

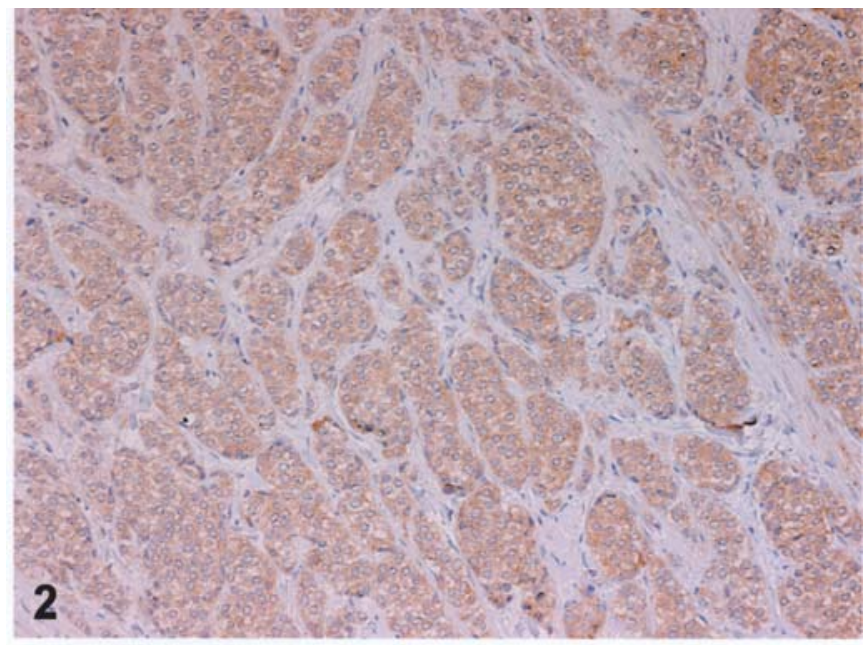

Figure 2. Synaptophysin is diffusely expressed in the tumor cells (case 4) (original magnification, $\mathrm{x} 100$ ).

\section{Results}

The clinicopathological and immunohistochemical findings from 12 appendiceal well-differentiated endocrine neoplasms are summarized in Table I. The median tumor size was $9.1 \mathrm{~mm}$ (range 2-20 mm) (Table I). All of the 12 tumors grew in the form of solid nests, trabeculae or pseudoglands and were comprised of monomorphous neoplastic cells showing mild or no atypia (Fig. 1). These tumors frequently involved muscularis propria, but were confined to the appendiceal wall, and mesoappendix involvement was not revealed. Angioinvasion and metastasis to lymph nodes or to other organs were also absent in all cases, and these findings satisfied the World Health Organization's criteria of well-differentiated endocrine neoplasms $(11,12)$.

Synaptophysin and chromogranin A were diffusely expressed in all cases (Figs. 2 and 3). Serotonin was frequently revealed in the tumor cells (10/12 cases) (Fig. 4). In addition, peripherin and $\alpha$-internexin were detected in all cases (12/12)

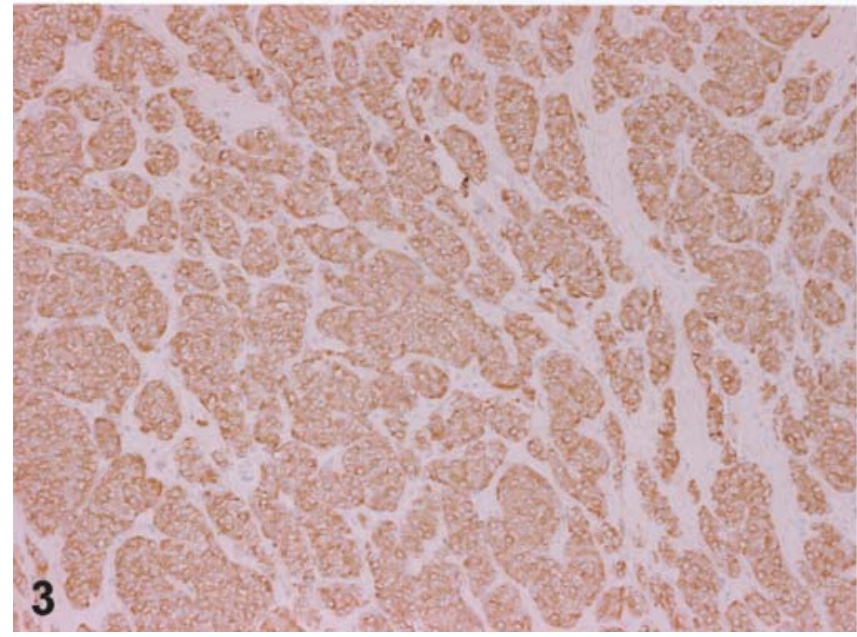

Figure 3. Chromogranin A is diffusely expressed in the tumor cells (case 4) (original magnification, $\mathrm{x} 100$ ).

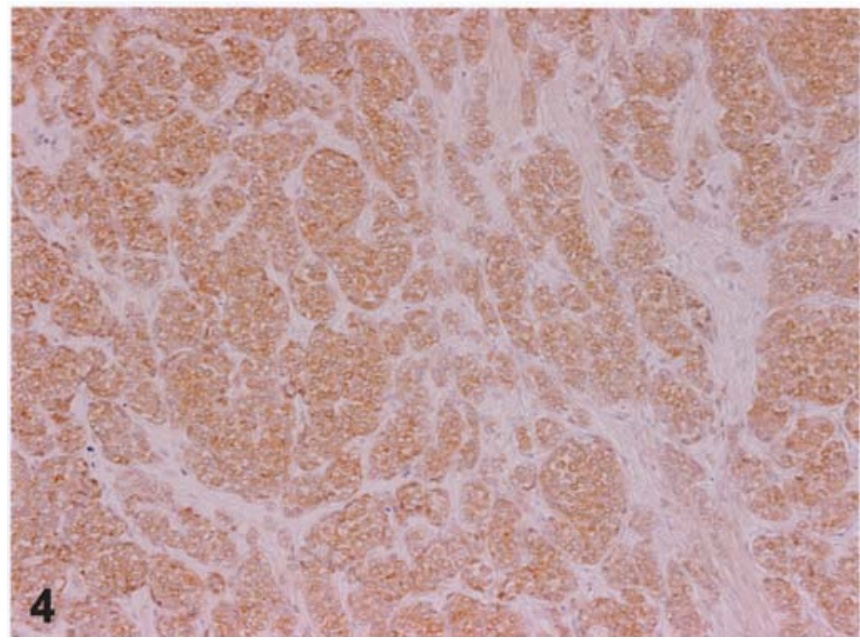

Figure 4. Serotonin is detected in most of the tumor cells (case 4) (original magnification, $\mathrm{x} 100)$.

(Figs. 5 and 6). Tumor cells positive for peripherin were abundant in all cases, and $\alpha$-internexin-positive tumor cells were abundant in 7 cases (Table I). These two neuronal intermediate filaments were co-expressed in a considerable number of tumor cells in all cases, but tumor cells that were positive for only one or the other of these intermediate filaments were also observed, especially in cases 5, 7-9 and 11. NCAM was detected in all cases (12/12) (Fig. 7). The Ki-67 labeling indices were $<1 \%$ in all cases (Fig. 8).

\section{Discussion}

The appendix and rectum are the two major sites of endocrine neoplasms in the lower gastrointestinal tract. However, appendiceal endocrine neoplasms are less frequent in Japan than in western countries (13), and there was only one Japanese case out of the 12 . The appendix arises from the distal portion of the midgut, and the well-differentiated endocrine neoplasms located in it frequently exhibit enterochromaffin 


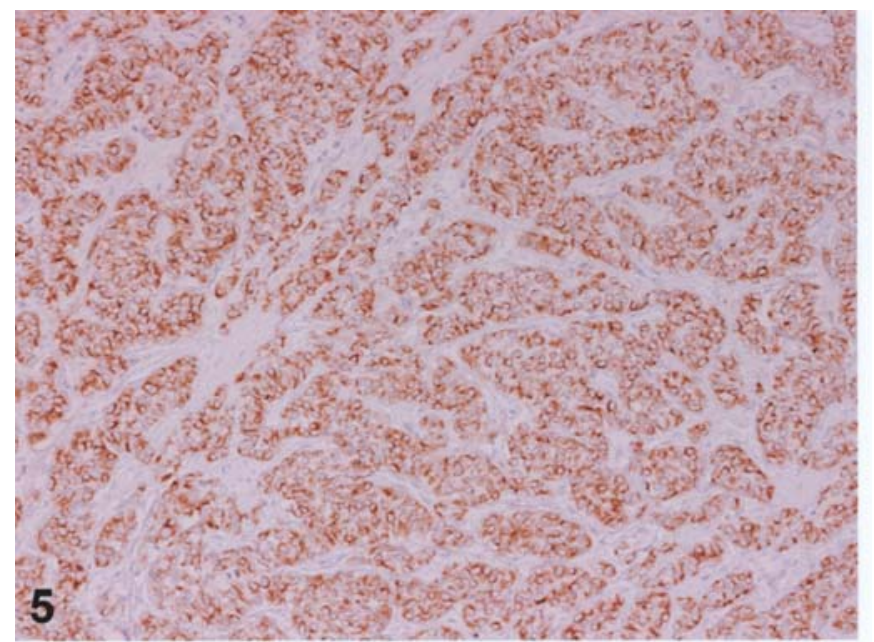

Figure 5. Peripherin is diffusely expressed in the tumor cells (case 4) (original magnification, $\mathrm{x} 100$ ).

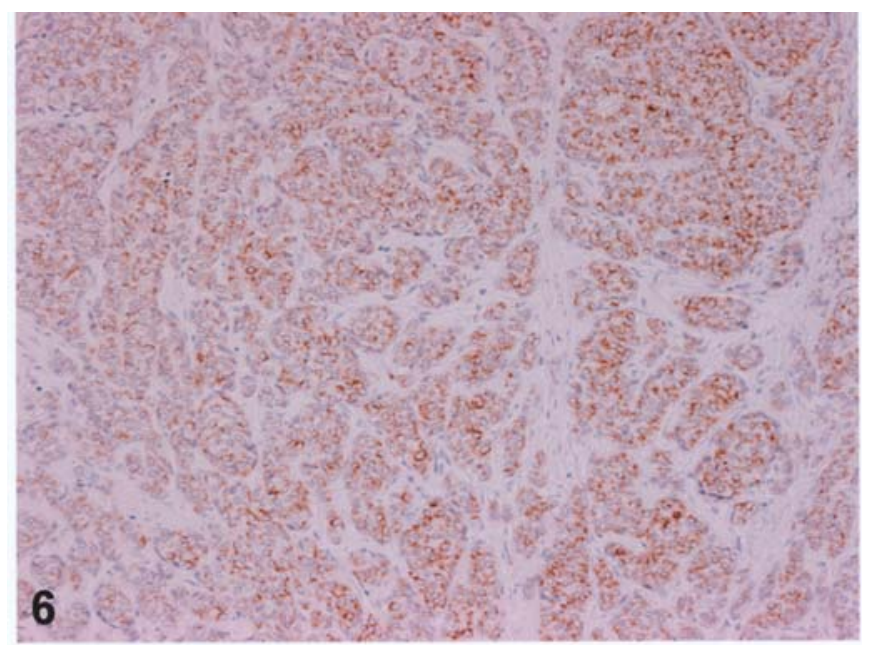

Figure 6. $\alpha$-internexin is detected in most of the tumor cells (case 4) (original magnification, $\mathrm{x} 100)$.

differentiation with serotonin synthesis, the most common phenotype of endocrine cells in this organ $(14,15)$. The high incidence of serotonin expression in our 12 cases (10/12) and the diffuse immunoreactivity to chromogranin A, a vesicular protein associated with amine-containing granules, in all our cases seems to be related to the high frequency of enterochromaffin differentiation. On the other hand, the rectum arises from the distal end of the hindgut and is rich in argyrophilic cells (L cells) with enteroglucagon or peptide YY (15). However, enterochromaffin differentiation is much less frequent in the rectum than in the appendix. This phenomenon seems to correlate with a lower incidence of immunoreactivity to serotonin and chromogranin $\mathrm{A}$ in well-differentiated endocrine neoplasms of the rectum than of the appendix, as shown in our previous study where positive immunoreactivity to both serotonin and chromogranin A was demonstrated in 5 of 12 rectal cases (10). Al-Khafaji et al reported that the incidence of NCAM immunoreactivity in midgut welldifferentiated endocrine neoplasms $(58 \%, 7 / 12$ cases $)$ was higher than in hindgut cases (20\%, 2/10 cases) (14). However,

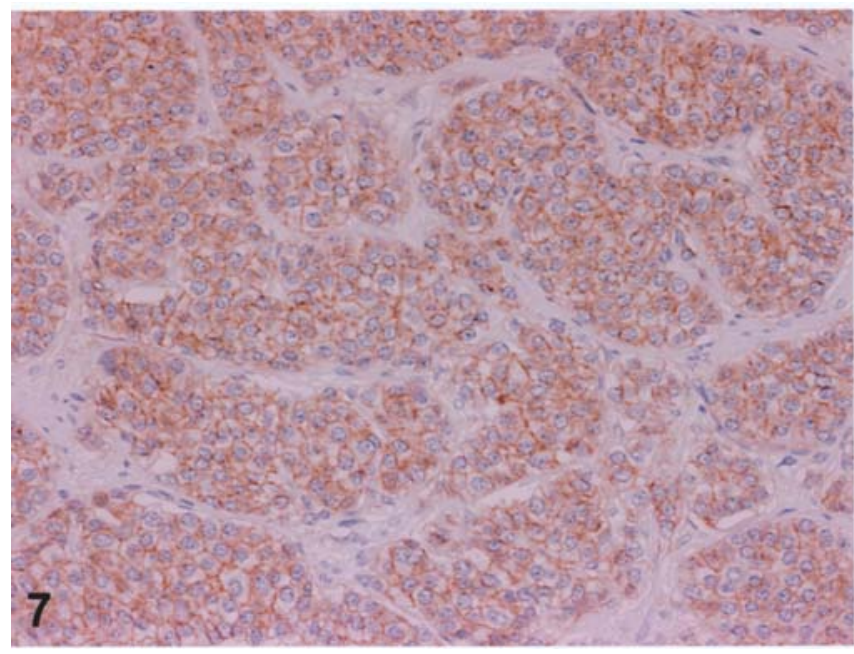

Figure 7. NCAM is expressed in the tumor cell membrane (case 4) (original magnification, $\mathrm{x} 200)$.

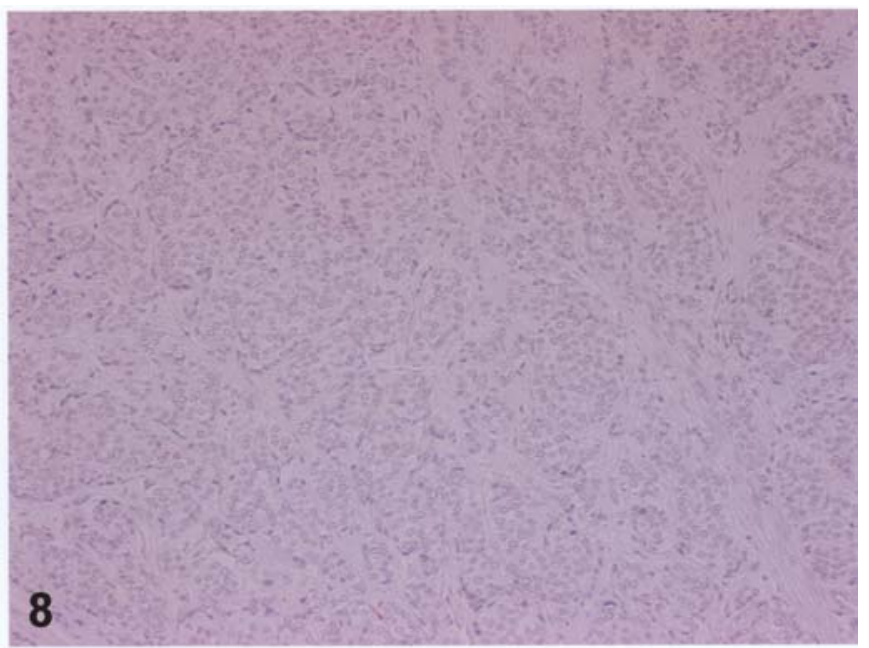

Figure 8 . Ki-67 labeling index is $<1 \%$ (case 4) (original magnification, $\mathrm{x} 100)$.

their midgut cases almost exclusively comprised small intestinal tumors (11/12 cases) and were therefore inappropriate for the evaluation of the incidence of NCAM reactivity in appendiceal tumors. Our present analyses revealed NCAM in all 12 appendiceal well-differentiated endocrine neoplasms $(12 / 12)$, an incidence much higher than that found in rectal tumors (14).

The well-differentiated endocrine neoplasms of visceral organs have several markers in common with neurons and epithelial markers. However, the expression of neuronal intermediate filaments had not been shown in these until our previous study demonstrated the consistent expression of peripherin in rectal well-differentiated endocrine neoplasms without metastases (10). Our present analyses revealed a consistent and diffuse expression of peripherin in the appendiceal well-differentiated endocrine neoplasms as well as in the rectal cases, and demonstrated that peripherin expression is a common feature in well-differentiated endocrine neoplasms of the lower gastrointestinal tract. In addition to peripherin, another neuronal intermediate filament, 
$\alpha$-internexin, was found in all 12 of these appendiceal welldifferentiated endocrine neoplasms. $\alpha$-internexin belongs to type IV intermediate filaments and is widely distributed in immature central neurons and in some peripheral neurons. It supposedly plays important roles in the development and maintenance of neurons $(3,4)$. This intermediate filament is frequently expressed in central nervous system tumors, such as medulloblastomas (7) and neuroblastomas (8), occasionally with the co-expression of peripherin $(7,8)$. However, the expression of $\alpha$-internexin had not been reported in gastrointestinal endocrine neoplasms prior to the present report. As described, all of our appendiceal well-differentiated endocrine neoplasms had $\alpha$-internexin-positive cells, which were quite abundant in 7 cases. $\alpha$-internexin and peripherin were coexpressed in all the appendiceal well-differentiated endocrine neoplasms examined.

Our preliminary study revealed sporadic expression of $\alpha$-internexin in some of the rectal well-differentiated endocrine neoplasms (4/8 cases) in addition to peripherin, but the frequency of positive cells was far less than that found in appendiceal tumors (unpublished data). To the best of our knowledge, immunohistochemical analyses of peripherin and $\alpha$-internexin expression have not been reported in welldifferentiated endocrine neoplasms of the upper gastrointestinal tract, respiratory tract or other visceral organs, so the exact distribution of these two neuronal intermediate filaments in well-differentiated endocrine neoplasms remains to be clarified.

In conclusion, peripherin was consistently expressed in all the appendiceal well-differentiated endocrine neoplasms. There was no difference in the manner of peripherin expression between appendiceal and rectal well-differentiated endocrine neoplasms. In addition, co-expression of peripherin and $\alpha$-internexin was demonstrated in all 12 of our appendiceal cases, and was quite abundant in 7 . On the other hand, incidences of immunoreactivity to NCAM and chromogranin $\mathrm{A}$ in appendiceal and rectal well-differentiated endocrine neoplasms differed considerably and were much higher in the appendiceal than the rectal cases. Peripherin expression is a common feature of appendiceal and rectal well-differentiated endocrine neoplasms, but the manner of neural marker expression is different and depends on the site of origin.

\section{Acknowledgements}

The authors wish to thank Mr. Y. Miyahira, Mr. M. Iwai and Ms. Y. Iwata for their technical assistance.

\section{References}

1. Escurat M, Djabali K, Gumpel M, Gros F and Portier MM: Differential expression of two neuronal intermediate-filament proteins, peripherin and the low-molecular-mass neurofilament protein (NF-L), during the development of the rat. J Neurosci 10: 764-784, 1990.

2. Fuchs E and Weber K: Intermediate filaments: structure, dynamics, function, and disease. Annu Rev Biochem 63: 345-382, 1994.

3. Fliegner KH, Kaplan MP, Wood TL, Pintar JE and Liem RK: Expression of the gene for the neuronal intermediate filament protein alpha-internexin coincides with the onset of neuronal differentiation in the developing rat nervous system. J Comp Neurol 342: 161-173, 1994.

4. Chien CL, Mason CA and Liem RK: Alpha-internexin is the only neuronal intermediate filament expressed in developing cerebellar granular neurons. J Neurobiol 29: 304-318, 1996.

5. Rauch U, Klotz M, Maas-Omlor S, et al: Expression of intermediate filament proteins and neuronal markers in the human fetal gut. J Histochem Cytochem 54: 39-46, 2006.

6. Pedersen WA, Becker LE and Yeger H: Expression and distribution of peripherin protein in human neuroblastoma cell lines. Int J Cancer 53: 463-470, 1993.

7. Kaya B, Mena H, Miettinen M and Rushing EJ: Alpha-internexin expression in medulloblastomas and atypical teratoid-rhabdoid tumors. Clin Neuropathol 22: 215-221, 2003.

8. Foley J, Witte D, Chiu FC and Parysek LM: Expression of the neural intermediate filament proteins peripherin and neurofilament-66/alpha-internexin in neuroblastoma. Lab Invest 71: 193-199, 1994.

9. Baudoin C, Meneguzzi G, Portier MM, et al: Peripherin, a neuronal intermediate protein, is stably expressed by neuroendocrine carcinomas of the skin, their xenograft on nude mice, and the corresponding primary cultures. Cancer Res 53: 1175-1181, 1993

10. Ishida M, Kushima R, Chano T and Okabe H: Immunohistochemical demonstration of the type III intermediate filament peripherin in human rectal mucosae and well-differentiated endocrine neoplasms. Oncol Rep 18: 633-637, 2007.

11. Capella C, Solcia E, Sobin LH and Arnold R: Endocrine tumors of the appendix. In: World Health Organization Classification of Tumors, Pathology and Genetics of Tumors of the Digestive Systems. Hamilton SR and Aaltonen LA (eds). IARC Press, Lyon, pp99-101, 2000.

12. Solcia E, Klöppel G and Sobin LH: Endocrine tumors of the gastrointestinal tract. In: Histological Typing of Endocrine Tumors. Solcia E, Klöppel G and Sobin LH (eds). 2nd edition, Springer-Verlag, Berlin, pp61-69, 2000.

13. Ito $\mathrm{T}$, Tanaka $\mathrm{M}$, Sasano $\mathrm{H}$, et al: Preliminary results of a Japanese nationwide survey of neuroendocrine gastrointestinal tumors. J Gastroenterol 42: 497-500, 2007.

14. A1-Khafaji B, Noffsinger AE, Miller MA, DeVoe G, Stemmermann GN and Fenoglio-Preiser C: Immunohistologic analysis of gastrointestinal and pulmonary carcinoid tumors. Hum Pathol 29: 992-999, 1998.

15. Williams GT: Endocrine tumors of the gastrointestinal tractselected topics. Histopathology 50: 30-41, 2007. 